\title{
Rho meson decay in the presence of a magnetic field
}

\author{
Aritra Bandyopadhyay ${ }^{\mathrm{a}}$ (D), S. Mallik ${ }^{\mathrm{b}}$ \\ Theory Division, Saha Institute of Nuclear Physics, HBNI, 1/AF Bidhannagar, Kolkata 700064, India
}

Received: 29 June 2017 / Accepted: 3 November 2017 / Published online: 16 November 2017

(C) The Author(s) 2017. This article is an open access publication

\begin{abstract}
We find a general expression for the one-loop self-energy function of neutral $\rho$-meson due to $\pi^{+} \pi^{-}$intermediate state in a background magnetic field. The pion propagator used in this expression was given by Schwinger; it is valid for arbitrary magnitudes of this field. Restricting our study to weak fields, we calculate the decay rate $\Gamma\left(\rho^{0} \rightarrow\right.$ $\pi^{+}+\pi^{-}$), which changes negligibly from the vacuum value.
\end{abstract}

\section{Introduction}

Though some stellar objects (like neutron stars) have long been known to possess magnetic fields [1-3], the realization that such fields are created in noncentral collisions of heavy ions [4-7] has initiated the search for effects of this background magnetic field on various observables [8-11]. Thus its effect on dilepton production [12-14] and on resonances created in the hadron phase [15-19] are investigated in detail. A more involved effect of this background field, called the chiral magnetic effect, demonstrates the topological nature of the QCD vacuum $[4,20,21]$. Apart from these effects in heavy ion collisions, the magnetic field can enhance the symmetry breaking of a theory, e.g. it increases the magnitude of the quark condensate, which breaks the flavor symmetry of QCD [22-24].

Here we investigate the effect of an external magnetic field in the decay of the $\rho$-meson in the dominant channel $\rho^{0} \rightarrow \pi^{+}+\pi^{-}$[15], which may affect the estimate of pion production in noncentral heavy ion collisions. This decay rate may be obtained from the imaginary part of the self-energy graph of the $\rho$-meson with a two-pion intermediate state (Fig. 1). The effect of the external field can be included in the decay process by taking the modified pion propagation in this field.

Such a modified (scalar and spinor) propagator in coordinate space has been derived long ago by Schwinger [25], to

\footnotetext{
a e-mail: aritra.bandyopadhyay@ saha.ac.in

be-mail: mallik@theory.saha.ernet.in
}

all orders in the external electromagnetic field, as an integral over proper time. Working in quantum electrodynamics, he used it to find corrections to the Maxwell Lagrangian. But in writing the electron self-energy function, he wrote the usual form, namely an integral over intermediate momentum $k_{\mu}$ with an electron propagator that depends on the kinematical momentum $\Pi_{\mu}$, containing the electromagnetic potential $A_{\mu}$ [26]. Then the shift of the origin in $k$ space, necessary in carrying out the $k$-integration, cannot be made, owing to the noncommutativity of the components of $\Pi_{\mu}$. He circumvented this difficulty by an ingenious $\xi$-device ${ }^{1}$ and evaluated the self-energy function analytically for weak and strong magnetic fields.

In this work we write the pion self-energy in coordinate space with pion propagators as given by Schwinger. There is no difficulty here as it contains no operators. The resulting expression is Fourier transformed to momentum space. Having obtained the $\rho^{0}$ self-energy with the pion propagator for a general external field, we find its decay rate in a weak magnetic field.

In Sect. 2 we outline Schwinger's derivation of the scalar propagator in coordinate space. In Sect. 3 we find the $\rho$ meson self-energy, first in a general background field and then specialized to a magnetic field. In Sect. 4 we calculate the decay rate to order quadratic in the magnetic field. Finally a general discussion of our method is given in the last Sect. 5. Appendix A evaluates the relevant integrals. Finally as a consistency check, in Appendix B we evaluate the vacuum decay rate of neutral $\rho$-meson.

\section{Scalar propagator}

The Lagrangian for the system of neutral $\rho$-meson and charged pions in the presence of an external electromagnetic field $A_{\mu}(x)$ is taken to be

$\overline{{ }^{1} \text { See Ref. [26] }}$, vol. II, p. 224; vol. III, p. 145. 


$$
\begin{aligned}
\mathcal{L}= & -\frac{1}{2}\left(\partial_{\mu} \rho_{\nu}-\partial_{\nu} \rho_{\mu}\right)^{2}+\frac{m_{\rho}^{2}}{2} \rho_{\mu} \rho^{\mu}+\left(D_{\mu} \phi\right)^{\dagger} D^{\mu} \phi \\
& -m^{2} \phi^{\dagger} \phi+i g \rho^{\mu} \phi^{\dagger} \overleftrightarrow{\partial_{\mu}} \phi
\end{aligned}
$$

with $\partial_{\mu} \rho^{\mu}=0, D^{\mu}=\partial^{\mu}+i e A^{\mu}$. Though gauge invariance requires the interaction vertex to depend on $A_{\mu}(x)$ (i.e. $\partial_{\mu} \rightarrow D_{\mu}$ ), we assume it to be independent of the field, considering its effect only on the propagation of charged pions. The coupling $g$ can be found from the experimental decay width $\Gamma\left(\rho^{0} \rightarrow \pi^{+} \pi^{-}\right)=149 \mathrm{MeV}$ in vacuum to give $g=6.0$.

The equation of motion for the pion field is given by

$$
\left[\left(\partial_{\mu}+i e A_{\mu}\right)\left(\partial^{\mu}+i e A^{\mu}\right)+m^{2}\right] \phi(x)=0
$$

and the pion propagator is defined as

$G\left(x, x^{\prime}\right)=i\left\langle 0\left|T \phi(x) \phi^{\dagger}\left(x^{\prime}\right)\right| 0\right\rangle$,

where $T$ represents the time ordering and $|0\rangle$ is the vacuum for the quantum fields. The propagator satisfies

$$
\left[\left(\partial_{\mu}^{x}+i e A_{\mu}\right)\left(\partial_{x}^{\mu}+i e A^{\mu}\right)+m^{2}\right] G\left(x, x^{\prime}\right)=\delta^{4}\left(x-x^{\prime}\right) .
$$

We now review the steps arising in Schwinger's derivation of the exact propagator. If we introduce states labeled by space-time coordinates, $G\left(x, x^{\prime}\right)$ may be written as the matrix element of an operator $G$

$G\left(x, x^{\prime}\right)=\left\langle x^{\prime}|G| x\right\rangle$,

when we can express Eq. (2.4) as an operator equation

$\left(-\Pi^{2}+m^{2}\right) G=1$

where $\Pi_{\mu}=p_{\mu}-e A_{\mu}, p_{\mu}=i \partial_{\mu}$, satisfying the commutation relations,

$$
\begin{aligned}
{\left[x^{\mu}, \Pi^{\nu}\right] } & =-i g^{\mu \nu}, \\
{\left[\Pi^{\mu}, \Pi^{\nu}\right] } & =-i e F^{\mu \nu}, \quad\left(F^{\mu \nu}=\partial^{\mu} A^{v}-\partial^{v} A^{\mu}\right) .
\end{aligned}
$$

The operator equation (2.6) has the formal solution

$G=\frac{1}{-\Pi^{2}+m^{2}}=i \int_{0}^{\infty} \mathrm{d} s U(s)$,

where ${ }^{2}$

$U(s)=e^{-i H s}, \quad H=-\Pi^{2}+m^{2}$.

This notation emphasizes that $U(s)$ may be regarded as the operator describing the dynamics of a particle governed by the Hamiltonian ' $H$ ' in the proper time parameter ' $s$ '. The

${ }^{2}$ Here $m^{2}$ is understood to be $m^{2}-i \epsilon$, the infinitesimal providing convergence to the $s$-integral and the boundary conditions for the time ordered propagator. spacetime coordinate $x^{\mu}=(t, \vec{x})$ of the particle depends on this parameter.

In the Heisenberg representation the operators $x_{\mu}$ and $\Pi_{\mu}$ have the 'time' dependence

$x_{\mu}(s)=U^{\dagger}(s) x_{\mu} U(s), \quad \Pi_{\mu}(s)=U^{\dagger}(s) \Pi_{\mu} U(s)$,

and the basis ket and bra evolve as

$\left|x^{\prime} ; s\right\rangle=U^{\dagger}(s)\left|x^{\prime}\right\rangle, \quad\left\langle x^{\prime} ; s\right|=\langle x| U(s)$.

Then the construction of $G\left(x^{\prime}, x\right)$ reduces to the evaluation of

$\left\langle x^{\prime \prime}|U(s)| x^{\prime}\right\rangle=\left\langle x^{\prime \prime} ; s \mid x^{\prime} ; 0\right\rangle$,

which is the transformation function from a state in which the position operator $x_{\mu}(s=0)$ has value $x_{\mu}^{\prime}$, to a state in which $x_{\mu}(s)$ has the value $x_{\mu}^{\prime \prime}$. The equations of motion for the operators following from Eq. (2.10) are

$\frac{\mathrm{d} x_{\mu}}{\mathrm{d} s}=-i\left[x_{\mu}, H\right], \quad \frac{\mathrm{d} \Pi_{\mu}}{\mathrm{d} s}=-i\left[\Pi_{\mu}, H\right]$.

The transformation function itself can be found by solving the differential equation satisfied by it,

$i \frac{\mathrm{d}}{\mathrm{d} s}\left\langle x^{\prime \prime} ; s \mid x^{\prime} ; 0\right\rangle=\left\langle x^{\prime \prime} ; s|H(x(s), \Pi(s))| x^{\prime} ; 0\right\rangle$,

and

$\left(i \partial_{\mu}^{x^{\prime \prime}}-e A_{\mu}\left(x^{\prime \prime}\right)\right)\left\langle x^{\prime \prime} ; s \mid x^{\prime} ; 0\right\rangle=\left\langle x^{\prime \prime} ; s\left|\Pi_{\mu}(s)\right| x^{\prime} ; 0\right\rangle$,

along with a similar one for $\Pi_{\mu}(0)$, with boundary conditions

$$
\left.\left\langle x^{\prime \prime} ; s \mid x^{\prime} ; 0\right\rangle\right|_{s \rightarrow 0}=\delta^{4}\left(x^{\prime \prime}-x^{\prime}\right), \quad \lim _{s \rightarrow \infty}\left\langle x^{\prime \prime} ; s \mid x^{\prime} ; 0\right\rangle=0 .
$$

We now specialize to constant field strength, for which Eq. (2.13) can be solved exactly. Then Eq. (2.13) becomes, considering matrix notation,

$\frac{\mathrm{d} x}{\mathrm{~d} s}=2 \Pi, \quad \frac{\mathrm{d} \Pi}{\mathrm{d} s}=2 e F \Pi$.

The second equation of (2.17) can be immediately solved to give

$\Pi(s)=e^{2 e F s} \Pi(0)$.

With this solution the first equation of (2.17) yields the solution

$x(s)-x(0)=\frac{e^{2 e F s}-1}{e F} \Pi(0)$.

Using (2.18) and (2.19) and the antisymmetry of the field tensor $\left(F^{\mu \nu}=-F^{v \mu}\right)$ we get

$$
\begin{aligned}
\Pi^{2}(s) & =(x(s)-x(0)) K(x(s)-x(0)), \\
K & =\frac{e^{2} F^{2}}{4}[\sinh (e F s)]^{-2} .
\end{aligned}
$$


To evaluate the matrix element on the right of Eq. (2.14), we need to order the operator $x(s)$ to the left of $x(0)$, which would require the commutator

$$
\left[x_{\mu}(s), x_{\nu}(0)\right]=i\left(\frac{e^{2 e F s}-1}{e F}\right)_{\mu \nu} .
$$

Then we get

$$
\left\langle x^{\prime \prime} ; s|H(s)| x^{\prime} ; 0\right\rangle=f\left(x^{\prime \prime}, x^{\prime} ; s\right)\left\langle x^{\prime \prime} ; s \mid x^{\prime} ; 0\right\rangle,
$$

where

$$
f=-\left(x^{\prime \prime}-x^{\prime}\right) K\left(x^{\prime \prime}-x^{\prime}\right)-\frac{i}{2} \operatorname{tr}[e F \operatorname{coth}(e F s)]+m^{2},
$$

with tr indicating the trace over $4 \times 4$ matrices. Equation (2.14) can now be solved,

$$
\begin{aligned}
\left\langle x^{\prime \prime} ; s \mid x^{\prime} ; 0\right\rangle= & C\left(x^{\prime \prime}, x^{\prime}\right) \exp \left[-i \int \mathrm{d} s^{\prime} f\left(x^{\prime \prime}, x^{\prime} ; s\right)\right] \\
= & C\left(x^{\prime \prime}, x^{\prime}\right) \frac{1}{s^{2}} e^{-L(s)} \exp \left[-\frac{i}{4}\left(x^{\prime \prime}-x^{\prime}\right) R(s)\left(x^{\prime \prime}-x^{\prime}\right)\right. \\
& \left.-i\left(m^{2}-i \epsilon\right) s\right],
\end{aligned}
$$

where ${ }^{3}$

$L(s)=\frac{1}{2} \operatorname{tr} \ln \left[(e F s)^{-1} \sinh (e F s)\right]$,

$R(s)=e F \operatorname{coth}(e F s)$.

The $s$-independent function $C$ can be found by solving Eq. (2.15),

$C\left(x^{\prime \prime}, x^{\prime}\right)=C\left(x^{\prime \prime}\right) \exp \left[-i e \int_{x^{\prime}}^{x^{\prime \prime}} \mathrm{d} \xi\left[A(\xi)+\frac{1}{2} F\left(\xi-x^{\prime}\right)\right]\right]$.

As $A(\xi)+\frac{1}{2} F\left(\xi-x^{\prime}\right)$ has vanishing curl, it can be written as

$C\left(x^{\prime \prime}, x^{\prime}\right)=C \Phi\left(x^{\prime \prime}, x^{\prime}\right)$

$\Phi\left(x^{\prime \prime}, x^{\prime}\right)=\exp \left[-i e \int_{x^{\prime}}^{x^{\prime \prime}} \mathrm{d} \xi A(\xi)\right]$,

where the integration in the phase factor $\Phi$ runs on a straight line between $x^{\prime}$ and $x^{\prime \prime}$ and the $F$ term vanishes. The constant $C$ is given by the first boundary condition of (2.16) as

$1=\frac{C}{s^{2}} \int \mathrm{d}^{4} x \exp \left(-\frac{i}{4 s} x^{2}\right)$.

\footnotetext{
$\overline{3}$ In writing $L(s)$ we follow Schwinger in choosing the integration constant to make $C$ independent of external field and also in extracting the coordinate independent, singular $s$ behavior.
}

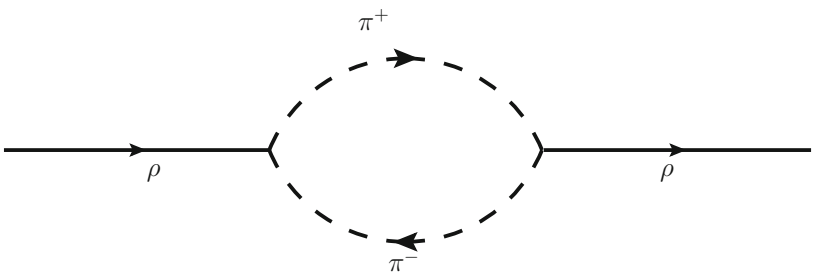

Fig. 1 Self-energy graph of the $\rho$-meson from a two-pion intermediate state in the presence of a magnetic field of arbitrary strength

This integral is evaluated in Appendix A to give $C=$ $-i /(4 \pi)^{2}$.

We finally get the transformation function as

$$
\begin{aligned}
\left\langle x^{\prime \prime} ; s \mid x^{\prime} ; 0\right\rangle= & -\frac{i}{(4 \pi)^{2} s^{2}} \Phi\left(x^{\prime \prime}, x^{\prime}\right) e^{-L(s)} \\
& \times \exp \left[-\frac{i}{4}\left(x^{\prime \prime}-x^{\prime}\right) R(s)\left(x^{\prime \prime}-x^{\prime}\right)\right. \\
& \left.-i\left(m^{2}-i \epsilon\right) s\right]
\end{aligned}
$$

which in turn gives the propagator

$G\left(x^{\prime \prime}, x^{\prime}\right)=i \int_{0}^{\infty} \mathrm{d} s\left\langle x^{\prime \prime} ; s \mid x^{\prime} ; 0\right\rangle$.

In our work below we shall encounter the product of propagators, $G\left(x^{\prime}, x^{\prime \prime}\right) G\left(x^{\prime \prime}, x^{\prime}\right)$ with derivatives acting on them. Without the derivatives, the phase factors in $G$ would cancel out mutually. In the presence of derivatives we can still get rid of the phase factors, if we make a gauge choice in the potential, replacing $A_{\mu}$ with

$A_{\mu}^{\prime}(x)=A_{\mu}(x)-\partial_{\mu} \lambda(x), \quad \lambda(x)=\int_{x^{\prime}}^{x} \mathrm{~d} \xi^{\mu} A_{\mu}(\xi)$,

when Eq. (2.4) is satisfied by $G$ without the phase factor $\Phi .{ }^{4}$ In the following we choose this gauge to write $G\left(x^{\prime \prime}, x^{\prime}\right)$ without this phase.

\section{$3 \rho$ self-energy in external field}

We now express the self-energy graph of Fig. 1 in terms of the pion propagators in external field given by Eqs. (2.29) and (2.30). We first keep the external (constant) field a general one, specializing later to the interesting case of pure magnetic field.

\footnotetext{
${ }^{4}$ See Ref. [26], vol. I, p. 271.
} 


\subsection{General field}

We now work out the complete $\rho$-propagator,

$D_{\lambda \sigma}^{\prime}\left(z, z^{\prime}\right)=i\left\langle 0\left|T \rho_{\lambda}(z) \rho_{\sigma}\left(z^{\prime}\right) \exp \left(i \int \mathrm{d} x \mathcal{L}_{\text {int }}(x)\right)\right| 0\right\rangle$,

to order $g^{2}$ in the interaction representation. We take the $\rho$ field as free, ${ }^{5}$ but the pion field lives in the background electromagnetic field. Contracting the fields for the graph of Fig. 1, we get

$$
\begin{aligned}
& D_{\lambda \sigma}^{\prime}\left(z-z^{\prime}\right)=D_{\lambda \sigma}\left(z-z^{\prime}\right) \\
& \quad+\int \mathrm{d}^{4} x^{\prime} \mathrm{d}^{4} x^{\prime \prime} D_{\lambda \mu}\left(z-x^{\prime}\right) \Sigma^{\mu \nu}\left(x^{\prime}-x^{\prime \prime}\right) D_{\nu \sigma}\left(x^{\prime \prime}-z\right),
\end{aligned}
$$

where $\Sigma$ is the self-energy tensor involving the two pion propagators. The propagators are distinguished only by their proper times $s_{1}$ and $s_{2}$, over which they are integrated. Carrying out the derivatives contained in $\mathcal{L}_{\text {int }}$ on these propagators, we get $[27,28]$

$$
\Sigma^{\mu \nu}(x)=-i\left(\frac{g}{32 \pi^{2}}\right)^{2} \int \frac{\mathrm{d} s_{1} \mathrm{~d} s_{2}}{s_{1}^{2} s_{2}^{2}} \exp \left[-L_{1}-L_{2}\right] \Lambda^{\mu \nu}(x)
$$

with

$$
\begin{aligned}
\Lambda^{\mu \nu}(x)= & {\left[x_{\alpha}\left(R_{1}-R_{2}\right)^{\alpha \mu}\left(R_{1}-R_{2}\right)^{\nu \beta} x_{\beta}+2 i\left(R_{1}+R_{2}\right)^{\mu \nu}\right] } \\
& \times \exp \left[-\frac{i}{4} x_{\alpha}\left(R_{1}+R_{2}\right)^{\alpha \beta} x_{\beta}-i m^{2}\left(s_{1}+s_{2}\right)\right] .
\end{aligned}
$$

Here $L_{1}=L\left(s_{1}\right), L_{2}=L\left(s_{2}\right)$ and similarly for $R_{1}, R_{2}$.

Having obtained Eq. (3.1) in configuration space, we go over to momentum space by taking Fourier transforms. Letting $K_{\mu \nu}(x)$ denote any of the $D_{\mu \nu}(x), D_{\mu \nu}^{\prime}(x)$ and $\Sigma_{\mu \nu}(x)$, their Fourier transforms are defined as

$$
K_{\mu \nu}(x)=\int \frac{\mathrm{d}^{4} q}{(2 \pi)^{4}} e^{-i q \cdot x} K_{\mu \nu}(q)
$$

and Eq. (3.1) becomes

$$
D_{\lambda \sigma}^{\prime}(q)=D_{\lambda \sigma}(q)+D_{\lambda \mu}(q) \Sigma^{\mu \nu}(q) D_{\nu \sigma}(q) \text {. }
$$

The vacuum and the complete propagator of the $\rho$-meson are given by

$$
\begin{aligned}
D_{\lambda \sigma}(q) & =\left(-g_{\lambda \sigma}+\frac{q_{\lambda} q_{\sigma}}{m^{2}}\right) D\left(q^{2}\right), \\
D\left(q^{2}\right) & =\frac{-1}{q^{2}-m_{\rho}^{2}+i \epsilon}, \\
D_{\lambda \sigma}^{\prime}(q) & =\left(-g_{\lambda \sigma}+\frac{q \lambda q_{\sigma}}{m^{2}}\right) D^{\prime}\left(q^{2}\right),
\end{aligned}
$$

5 The charged $\rho$-meson would acquire an external field dependent mass [15], if the field is large. But we do not include it here, as we are interested in the imaginary part of the self-energy. where $D^{\prime}\left(q^{2}\right)$ is the function we want to find.

A simplification results on noting that the $\rho$ field is coupled to a conserved pion current in the interaction lagrangian shown in Eq. (2.1). As a result, contracting $q_{\mu}$ and $q_{\nu}$ of the $\rho$ propagators with $\Sigma^{\mu v}$ in the second term of Eq. (3.4) yields zero. We are then left with the metric tensor in $\rho$ propagator. Contracting further the indices $\sigma$ and $\lambda$ we get

$$
-3 D^{\prime}\left(q^{2}\right)=-3 D\left(q^{2}\right)+D\left(q^{2}\right) \Sigma(q) D\left(q^{2}\right) .
$$

Here $\Sigma(q)$ is the Fourier transform of $\Sigma^{\mu v}(x)$, after contracting the indices,

$\Sigma(q)=-i\left(\frac{g}{32 \pi^{2}}\right)^{2} \int \frac{\mathrm{d} s_{1} \mathrm{~d} s_{2}}{s_{1}^{2} s_{2}^{2}} \exp \left[-L_{1}-L_{2}\right] \Lambda(q)$

with

$$
\begin{aligned}
\Lambda(q)= & \exp \left[-i m^{2}\left(s_{1}+s_{2}\right)\right] \\
& \int \mathrm{d}^{4} x\left[x^{\rho}\left(R_{1}-R_{2}\right)_{\rho \sigma}^{2} x^{\sigma}+2 i g_{\mu \nu}\left(R_{1}+R_{2}\right)^{\mu \nu}\right] \\
& \times \exp \left[i q \cdot x-\frac{i}{4} x^{\alpha}\left(R_{1}+R_{2}\right)_{\alpha \beta} x^{\beta}-i m^{2}\left(s_{1}+s_{2}\right)\right] .
\end{aligned}
$$

Clearly the expression for $\Sigma$ is divergent, to which we have to add renormalization counterterms. Beside canceling the divergent pieces, we shall choose the finite pieces in the counterterms such that the total self-energy $\Sigma_{t o t}$ satisfies

$$
\Sigma_{\text {tot }}\left(q^{2}=m_{\rho}^{2}\right)=0 ;\left.\quad \frac{\mathrm{d} \Sigma_{t o t}(q)}{\mathrm{d} q}\right|_{q^{2}=m_{\rho}^{2}}=0 .
$$

Then $m_{\rho}$ will remain the physical $\rho$-meson mass and $g$ the renormalized $\rho \pi \pi$ coupling. We shall come back to this renormalization in Sect. 4.

Including the sum of all reducible graphs in Eq. (3.7) we get the Dyson-Schwinger equation for the $\rho$ propagator:

$D^{\prime}\left(q^{2}\right)=D\left(q^{2}\right)-\frac{1}{3} D\left(q^{2}\right) \Sigma(q) D^{\prime}\left(q^{2}\right)$,

giving

$D^{\prime}\left(q^{2}\right)=\frac{-1}{q^{2}-m_{\rho}^{2}-\frac{1}{3} \Sigma(q)}$.

In the neighborhood of physical $\rho$-meson pole, it gives the decay width $\Gamma\left(\rho^{0} \rightarrow \pi^{+} \pi^{-}\right)$with background electromagnetic field:

$\Gamma=-\frac{\operatorname{Im} \Sigma}{3 m_{\rho}}$.

Below we shall calculate $\operatorname{Im} \Sigma$. 


\subsection{Pure magnetic field}

We derived above the expressions for the pion propagator and the consequent $\rho$-meson self-energy in a general background external field $F_{\mu \nu}$. We now specialize this field to a magnetic field $B$ in the $z$ direction, i.e. $F^{21}=-F^{12}=B$ and all other components zero. We can diagonalize this $2 \times 2$ matrix, in which only the coordinates $x^{\prime 1}$ and $x^{\prime 2}$ are involved. After diagonalization, the quantities involving matrices reduce to

$$
\begin{aligned}
& L(s)=\ln \left[(e B s)^{-1} \sin (e B s)\right], \\
& R=2 e B \cot (e B s)+\frac{2}{s} \\
& \left(x^{\prime \prime}-x^{\prime}\right) R(s)\left(x^{\prime \prime}-x^{\prime}\right)=-\left(x^{\prime \prime}-x^{\prime}\right)_{\perp}^{2} e B \cot (e B s) \\
& +\left(x^{\prime \prime}-x^{\prime}\right)_{\Perp}^{2} \frac{1}{s},
\end{aligned}
$$

where $\left(x_{\perp}^{\prime}\right)^{2}=\left(x^{\prime 1}\right)^{2}+\left(x^{\prime 2}\right)^{2} ;\left(x_{\| 1}^{\prime}\right)^{2}=\left(x^{\prime} 0\right)^{2}-\left(x^{\prime 3}\right)^{2}$. With these values, Eqs. (2.29) and (2.30) give the pion propagator in the magnetic field as

$$
\begin{aligned}
G(x)= & \frac{e B}{(4 \pi)^{2}} \int_{0}^{\infty} \frac{\mathrm{d} s}{s \sin (e B s)} \exp \left[i x_{\perp}^{2} \frac{e B}{4} \cot (e B s)\right. \\
& \left.-\frac{i}{4 s} x_{\|}^{2}-i\left(m^{2}-i \epsilon\right) s\right]
\end{aligned}
$$

Then the corresponding $\rho$ self-energy in momentum space can be obtained from Eqs. (3.8) and (3.9). It will involve two integrals over the proper time $s_{1}$ and $s_{2}$ of the propagating pions. We change these variables to $s$ and $u$ defined by

$s_{1}=s u, \quad s_{2}=s(1-u), \quad \mathrm{d} s_{1} \mathrm{~d} s_{2}=s \mathrm{~d} s \mathrm{~d} u$.

Introducing for short

$$
\begin{aligned}
\alpha & =(e B)^{2}[\cot (e B s u)-\cot (e B s(1-u))]^{2}, \\
\beta & =\frac{1}{s^{2}}\left(\frac{1}{u}-\frac{1}{(1-u)}\right)^{2}, \\
\gamma & =\frac{e B}{4}[\cot (e B s u)+\cot (e B s(1-u))], \\
\delta & =\frac{1}{4 s u(1-u)},
\end{aligned}
$$

we can now write the self-energy as

$$
\begin{aligned}
\Sigma\left(q_{\perp}, q_{\|}\right)= & -i\left(\frac{g}{32 \pi^{2}}\right)^{2} \int_{0}^{\infty} \frac{\mathrm{d} s}{s} \\
& \times \int_{0}^{1} \frac{\mathrm{d} u}{u(1-u)} \frac{(e B)^{2} \Lambda(s, u)}{\sin (e B s u) \sin (e B s(1-u))},
\end{aligned}
$$

with

$$
\begin{aligned}
\Lambda\left(s, u, q_{\|}, q_{\perp}\right)= & e^{-i m^{2} s} \int \mathrm{d}^{4} x e^{i q \cdot x}\left[-\alpha x_{\perp}^{2}+\beta x_{\|}^{2}\right. \\
& +16 i(\gamma+\delta)] \exp \left(i \gamma x_{\perp}^{2}-i \delta x_{\|}^{2}\right)
\end{aligned}
$$

which is evaluated in Appendix A, yielding

$$
\begin{aligned}
\Lambda(s, u)= & \frac{\pi^{2}}{\gamma \delta}\left[\frac{\alpha}{\gamma}\left(1-\frac{i q_{\perp}^{2}}{4 \gamma}\right)+\frac{\beta}{\delta}\left(1+\frac{i q_{\|}^{2}}{4 \delta}\right)-16(\gamma+\delta)\right] \\
& \times \exp \left[-i \frac{q_{\perp}^{2}}{4 \gamma}-i\left\{m^{2}-q_{\|}^{2} u(1-u)-i \epsilon\right\} s\right] .
\end{aligned}
$$

Note that all the parameters $\alpha, \beta, \gamma$ and $\delta$ are positive.

The $i \epsilon$ prescription in Eq. (3.18) makes the integration line in the $s$-plane run infinitesimally below the real axis, avoiding the singularities of the integrand. Then we can get rid of oscillations in it by deforming the integration line. As $u(1-$ $u) \leq 1 / 4$ for $0<u<1$, the quantity $\left[m^{2}-q_{\|}^{2} u(1-u)\right]$ appearing in Eq. (3.18) is positive, if we take $q_{\|}^{2}<4 m^{2}$, precluding physical pion pair creation. For such values of $q_{\|}^{2}$ we take a closed contour in the fourth quadrant, with vanishing contribution from the quarter circle. ${ }^{6}$ Changing the integration variable $s=-i t$ on the imaginary axis, we get

$$
\begin{aligned}
\Sigma\left(q_{\perp}, q_{\Perp}\right)= & \left(\frac{g}{32 \pi}\right)^{2} \int_{0}^{\infty} \frac{\mathrm{d} t}{t} \\
& \times \int_{0}^{1} \frac{\mathrm{d} u}{u(1-u)} \frac{(e B)^{2} \Lambda^{\prime}(t, u)}{\sinh (e B t u) \sinh (e B t(1-u))},
\end{aligned}
$$

where $\Lambda^{\prime}$ is related to $\Lambda$ by the change of variable. To write $\Lambda^{\prime}$, we define a new set of variables from Eq. (3.16),

$$
\begin{aligned}
\alpha^{\prime} & =(e B)^{2}[\operatorname{coth}(e B t u)-\operatorname{coth}(e B t(1-u))]^{2}, \\
\beta^{\prime} & =\frac{1}{t^{2}} \frac{(1-2 u)^{2}}{u^{2}(1-u)^{2}}, \\
\gamma^{\prime} & =\frac{e B}{4}[\operatorname{coth}(e B t u)+\operatorname{coth}(e B t(1-u))], \\
\delta^{\prime} & =\frac{1}{4 t u(1-u)} .
\end{aligned}
$$

\footnotetext{
${ }^{6}$ See Ref. [29], p. 322.
} 
In terms of these variables, we have

$$
\begin{aligned}
\Lambda^{\prime}= & \frac{1}{\gamma^{\prime} \delta^{\prime}}\left[\frac{\alpha^{\prime}}{\gamma^{\prime}}\left(1-\frac{q_{\perp}^{2}}{4 \gamma^{\prime}}\right)+\frac{\beta^{\prime}}{\delta^{\prime}}\left(1+\frac{q_{\|}^{2}}{4 \delta^{\prime}}\right)-16\left(\gamma^{\prime}+\delta^{\prime}\right)\right] \\
& \times \exp \left[-\frac{q_{\perp}^{2}}{4 \gamma^{\prime}}-\left\{m^{2}-q_{\|}^{2} u(1-u)\right\} t\right] .
\end{aligned}
$$

Another form of $\Sigma$, which will be useful for the discussion below may be obtained by scaling $t$ with $e B$, that is, we set $\bar{t}=e B t$, when $\Sigma$ becomes

$$
\Sigma=\int_{0}^{\infty} \frac{\mathrm{d} \bar{t}}{\bar{t}} \int_{0}^{1} \frac{\mathrm{d} u}{u(1-u)} \frac{\bar{\Lambda}}{\sinh (\bar{t} u) \sinh (\bar{t}(1-u))},
$$

where

$$
\begin{aligned}
\bar{\Lambda}= & \frac{1}{\bar{\gamma} \bar{\delta}}\left[\frac{\bar{\alpha}}{\bar{\gamma}}\left(e B-\frac{q_{\perp}^{2}}{4 \bar{\gamma}}\right)+\frac{\bar{\beta}}{\bar{\delta}}\left(e B+\frac{q_{11}^{2}}{4 \bar{\delta}}\right)-16 e B(\bar{\gamma}+\bar{\delta})\right] \\
& \times \exp \left[-\frac{q_{\perp}^{2}}{4 \bar{\gamma}}-\left\{\frac{m^{2}}{e B}-\frac{q^{2}}{e B} u(1-u)\right\} t\right]
\end{aligned}
$$

with

$$
\begin{array}{ll}
\bar{\alpha}=[\cot (\bar{t} u)-\cot (\bar{t}(1-u))]^{2}, & \bar{\beta}=\frac{1}{\bar{t}^{2}}\left(\frac{1}{u}-\frac{1}{(1-u)}\right)^{2}, \\
\bar{\gamma}=\frac{1}{4}[\cot (\bar{t} u)+\cot (\bar{t}(1-u))], \quad \bar{\delta}=\frac{1}{4 \bar{t} u(1-u)} .
\end{array}
$$

\section{$4 \rho$-meson decay}

As already stated, the self-energy function in Eq. (3.20) is valid for momenta for which the $\rho$-meson cannot decay into a pion pair. To calculate this decay rate we therefore need to continue Eq. (3.20) beyond such momenta [29]. This process of analytic continuation is immediate, if we can evaluate the $t$-integral analytically. However, the exact expression of Eq. (3.23) for $\Sigma$ containing various hyperbolic functions, making it difficult to do so. The procedure here is to consider separately weak $\left(e B<m^{2}\right)$ and strong $\left(e B>m^{2}\right)$ fields. ${ }^{7}$ As strong fields are considered extensively in the literature [1518], we take up the case of weak fields, which is realized, in particular, in the hadronic phase of noncentral heavy ion collisions. In this case the exponential in Eq. (3.24) shows that only correspondingly small values of $t$ can contribute. We can then expand the different functions in powers of $t$. To get the leading effect, we need to keep only the first two terms in their expansions. After some algebra, we get the self-energy as

\footnotetext{
$\overline{{ }^{7} \text { See Ref. [26] }}$, vol. III, p. 161-163.
}

$$
\begin{aligned}
\Sigma\left(q^{2}\right)= & \left(\frac{g}{2 \pi}\right)^{2} \frac{1}{2} \int_{0}^{\infty} \mathrm{d} t \int_{-1}^{1} \mathrm{~d} v\left(D+\frac{(e B)^{2}}{6} F\right) \\
& \times \exp \left[-\left\{m^{2}-\frac{q^{2}}{4}\left(1-v^{2}\right)\right\} t\right]
\end{aligned}
$$

where

$$
\begin{aligned}
& D=\frac{v^{2}}{t} \frac{q^{2}}{4}-\frac{2}{t^{2}}, \\
& F=\frac{3}{2}\left(1-v^{2}\right)-\frac{v^{2}}{4}\left\{q^{2}-2\left(1-v^{2}\right) q_{\perp}^{2}\right\} t .
\end{aligned}
$$

Here a numerical factor of $4^{4}$ has been put into the coupling constant factor. Also, for convenience, the variable $u$ is replaced by $\frac{1}{2}(1+v)$. The terms in $D$, containing divergences, are free of a magnetic field. We treat them in Appendix $\mathrm{B}$ to isolate their finite pieces and find the decay rate in vacuum.

The $e B$ dependent self-energy given by the $F$ terms in Eq. (4.2) can be rewritten as

$\Sigma_{e B}\left(q^{2}\right)=\left(\frac{g}{2 \pi}\right)^{2} \frac{(e B)^{2}}{6} \bar{\Sigma}\left(q^{2}\right)$

where

$$
\begin{aligned}
\bar{\Sigma}\left(q^{2}\right)= & \int_{0}^{1} \mathrm{~d} v\left[\frac{3}{2}\left(1-v^{2}\right)+\frac{v^{2}}{4}\left\{q^{2}-2\left(1-v^{2}\right) q_{\perp}^{2}\right\} \frac{\partial}{\partial m^{2}}\right] \\
& \times \int_{0}^{\infty} \mathrm{d} t \exp \left[-\left\{m^{2}-\frac{q^{2}}{4}\left(1-v^{2}\right)\right\} t\right] .
\end{aligned}
$$

We are now in a position to carry out the analytic continuation mentioned at the beginning of the section. If we hold the $q^{2}$ variable in the region $q^{2}<4 m^{2}$, the $t$-integration is well defined and can be integrated trivially to give

$$
\begin{aligned}
\bar{\Sigma}\left(q^{2}\right)= & \frac{3}{2} \int_{0}^{1} \mathrm{~d} v \frac{1-v^{2}}{m^{2}-q^{2}\left(1-v^{2}\right) / 4} \\
& +\frac{\partial}{\partial m^{2}} \int_{0}^{1} \mathrm{~d} v\left[\frac{v^{2}}{4}\left\{q^{2}-2\left(1-v^{2}\right) q_{\perp}^{2}\right\}\right] \\
& \times \frac{1}{m^{2}-q^{2}\left(1-v^{2}\right) / 4} .
\end{aligned}
$$

It can now be continued for $q^{2}<4 m^{2}$ in the $q^{2}$ plane with a cut along the real axis for $4 m^{2}<q^{2}<\infty$. To display this cut structure explicitly, we write $\bar{\Sigma}\left(q^{2}\right)$ as a dispersion integral by changing the integration variable $v$ to $q^{\prime 2}$ given by $v=\sqrt{1-4 m^{2} / q^{\prime 2}}$, getting 


$$
\begin{aligned}
\bar{\Sigma}\left(q^{2}\right)= & \int_{4 m^{2}}^{\infty} \frac{\mathrm{d} q^{\prime 2}}{q^{\prime 2}} \frac{1}{q^{\prime 2}-q^{2}}\left(1+\frac{12 m^{2}}{q^{\prime 2}}\right)\left(1-\frac{4 m^{2}}{q^{\prime 2}}\right)^{-1 / 2} \\
& +\frac{1}{2} \frac{\partial}{\partial m^{2}} \int_{4 m^{2}}^{\infty} \frac{\mathrm{d} q^{\prime 2}}{q^{\prime 2}} \frac{1}{q^{\prime 2}-q^{2}} \\
& \times\left(q^{2}-\frac{8 m^{2}}{q^{\prime 2}} q_{\perp}^{2}\right)\left(1-\frac{4 m^{2}}{q^{\prime 2}}\right)^{1 / 2} .
\end{aligned}
$$

Its imaginary part is given by the discontinuity across the cut,

$$
\begin{aligned}
\operatorname{Im} \bar{\Sigma}\left(q^{2}\right)= & \frac{1}{2 i}\left[\bar{\Sigma}\left(q^{2}+i \epsilon\right)-\bar{\Sigma}\left(q^{2}-i \epsilon\right)\right], \quad q^{2}>4 m^{2} \\
= & -\frac{\pi}{q^{2}}\left[\left(1-\frac{12 m^{2}}{q^{2}}-\frac{8 m^{2}}{q^{2}} \frac{q_{\perp}^{2}}{q^{2}}\right)\left(1-\frac{4 m^{2}}{q^{2}}\right)^{-1 / 2}\right. \\
& \left.+\frac{4 q_{\perp}^{2}}{q^{2}}\left(1-\frac{4 m^{2}}{q^{2}}\right)^{1 / 2}\right]
\end{aligned}
$$

From Eqs. (3.12), (4.3) and (4.7), we get the corresponding change in width as

$$
\begin{aligned}
\Gamma_{e B}= & \frac{g^{2}}{4 \pi} \frac{(e B)^{2}}{18 m_{\rho}^{3}}\left[1-10 \frac{m^{2}}{m_{\rho}^{2}}+4 \frac{q_{\perp}^{2}}{m_{\rho}^{2}}\left(1-\frac{4 m^{2}}{m_{\rho}^{2}}\right)\right. \\
& \left.+\mathcal{O}\left(\frac{m^{2}}{m_{\rho}^{2}}\right)^{2}\right] .
\end{aligned}
$$

Taking $m^{2} / m_{\rho}^{2}=1 / 30$, it becomes

$\Gamma_{e B}=\frac{g^{2}}{4 \pi} \frac{(e B)^{2}}{27 m_{\rho}^{3}}\left(1+\frac{26}{5} \frac{q_{\perp}^{2}}{m_{\rho}^{2}}\right)$.

For $e B<m^{2}$ and $q_{\perp}^{2}<m_{\rho}^{2}$, it gives $\Gamma_{e B}<0.6 \mathrm{MeV}$. The smallness of $\Gamma_{e B}$ may be explained by the fact that, while the (small) pion mass is the scale entering in the self-energy loop, it is evaluated at a (large) external momentum of $\rho$-meson mass. Also note that there is no pion mass in the denominator of Eq. (4.7). It is protected by chiral symmetry $(m \rightarrow 0)$, according to which physical quantities must be finite in this limit.

In passing, we note that the effect of the temperature on the decay width of the $\rho$-meson has been discussed extensively in the literature [30]. Here we have investigated the effect of weak magnetic fields on the same quantity and found it to be negligible compared to the thermal effects.

\section{Discussion}

In earlier calculations of hadron properties in a magnetic field [15-18], the majority of the references consider strong fields, taking the contribution of the leading Landau level for the system. A result to note at this point is that for strong enough fields the main decay channel, such as the $\rho^{0} \rightarrow$ $\pi^{+}+\pi^{-}$decay channel, which we are considering, may become closed. It is due to the generation of an effective pion mass $\bar{m}^{2}=m^{2}+e B$, causing the phase space for the process to shrink as the magnetic field becomes stronger [15].

In the present work we investigate the decay by setting up a general framework, valid for both weak and strong magnetic fields. It is obtained by writing the $\pi \pi$ loop in the correction to the $\rho$ propagator in configuration space, with the pion propagator as given by Schwinger [25]. When Fourier transformed, it gives the $\rho$-meson self-energy (3.20) as an integral over proper times, which is defined for momenta below the two-pion threshold.

If we now restrict the general representation Eq. (3.20) to weak fields $(e B<m)$, the exponential factor in it (or equivalently Eq. (3.24)) shows the leading contribution to arise from the neighborhood of proper time $t=0$, when we can expand the hyperbolic functions in powers of $t$. Still remaining below the two-pion threshold, we can integrate the resulting terms to get a series in powers of $(e B)^{2}$. These terms can be simply continued beyond the threshold and the imaginary part of the self-energy, so the decay width can be determined. In this work we retain only the $(e B)^{2}$ terms, though calculation of higher-order terms is also straightforward. As we show at the end of Sect. 4, the change in the decay width from the vacuum value turns out to be negligibly small.

So far we only discussed the effect of weak magnetic fields. But as already emphasized, strong field effects can also be obtained from the same general formula, Eq. (3.20). For $e B>m^{2}$, the exponential in this formula shows that large values of $t$ would also contribute. It is thus simple to keep the leading term in different hyperbolic functions. Collecting the exponentials in Eq. (3.20), we get

$\exp \left[-\frac{q_{\perp}^{2}}{2 e B}-\left\{m^{2}+e B-q_{\|}^{2} u(1-u)\right\} t\right]$,

giving the effective pion mass, as mentioned above [15-18].

There are at least two other methods of calculating the decay rate. One is Schwinger's $\xi$-device mentioned in Sect. 1 and the other is the Ritus method of an eigenfunction expansion [31]. It will be interesting to get comparable values from these methods.

Acknowledgements The work of $\mathrm{AB}$ is supported by the Department of Atomic Energy (DAE), India.

Open Access This article is distributed under the terms of the Creative Commons Attribution 4.0 International License (http://creativecomm ons.org/licenses/by/4.0/), which permits unrestricted use, distribution, and reproduction in any medium, provided you give appropriate credit to the original author(s) and the source, provide a link to the Creative Commons license, and indicate if changes were made.

Funded by SCOAP ${ }^{3}$. 


\section{Appendix A: Integrals}

Here we evaluate the integrals in Eqs. (2.28) and (3.18), paying attention to the phases appearing in the manipulations. First consider

$I=\int \mathrm{d}^{4} x e^{-i x^{2} / 4 s} \equiv J^{3} K, \quad s>0$,

where

$J=2 \int_{0}^{\infty} \mathrm{d} x_{1} e^{i x_{1}^{2} / 4 s}, \quad K=2 \int_{0}^{\infty} \mathrm{d} x_{0} e^{-i x_{0}^{2} / 4 s}$.

For $J$ we put $x_{1}^{2} / 4 s=u$ to get

$J=2 \sqrt{s} \int_{0}^{\infty} \mathrm{d} u u^{-1 / 2} e^{i u}$.

To avoid oscillations in the integrand, we take the contour of Fig. 2a in the first quadrant of the complex $u$ plane, so that the contribution from the quarter circle vanishes. As there is no singularity within and on the contour, the Cauchy formula gives

$J=-2 \sqrt{s} \int_{i \infty}^{0} \mathrm{~d} u u^{-1 / 2} e^{i u}$

If we now put $u=\exp (i \pi / 2) t$ on the integration line along the imaginary axis, we get

$J=2 \sqrt{s} e^{i \pi / 4} \int_{0}^{\infty} \mathrm{d} t t^{-1 / 2} e^{-t}=e^{i \pi / 4} \sqrt{4 \pi s}$,

the integral being the familiar Gamma function $\Gamma(1 / 2)=$ $\sqrt{\pi}$. The integral $K$ can be evaluated in the same way, taking the contour of Fig. 2 in the fourth quadrant, making the contribution of the quarter circle to vanish. We then get

$K=e^{-i \pi / 4} \sqrt{4 \pi s}$.

Putting the results (A.3) and (A.4) in (A.1) we get

$$
I=i(4 \pi s)^{2} .
$$

Next we consider the Fourier transform of Eq. (3.18),

$$
\begin{aligned}
\Lambda\left(s, u, q_{\|}, q_{\perp}\right)= & e^{-i m^{2} s} \int \mathrm{d}^{4} x e^{i q \cdot x}\left[-\alpha x_{\perp}^{2}+\beta x_{\|}^{2}\right. \\
& +16 i(\gamma+\delta)] \exp \left(i \gamma x_{\perp}^{2}-i \delta x_{\|}^{2}\right) .
\end{aligned}
$$

Here the basic integrals are

$$
\begin{aligned}
& L_{1}=\int_{-\infty}^{+\infty} \mathrm{d} x_{\perp}^{2} e^{-i q_{\perp} \cdot x_{\perp}+i \gamma x_{\perp}^{2}}, \\
& L_{2}=\int_{-\infty}^{+\infty} \mathrm{d} x_{0} e^{i q_{0} x_{0}-i \delta x_{0}^{2}}, \\
& L_{3}=\int_{-\infty}^{+\infty} \mathrm{d} x_{3} e^{-i q_{3} x_{3}+i \delta x_{3}^{2}}
\end{aligned}
$$

in terms of which the Fourier transform may be written as

$$
\begin{aligned}
\Lambda= & i \alpha \frac{\mathrm{d} L_{1}}{\mathrm{~d} \gamma} L_{2} L_{3}+i \beta\left(L_{1} \frac{\mathrm{d} L_{2}}{\mathrm{~d} \delta} L_{3}+L_{1} L_{2} \frac{\mathrm{d} L_{3}}{\mathrm{~d} \delta}\right) \\
& +16 i(\gamma+\delta) L_{1} L_{2} L_{3} .
\end{aligned}
$$

The basic integrals are generally of the same form as $J$ and $K$, if we complete the squares in the exponents. Thus

$$
\begin{aligned}
L_{1} & =e^{-i q_{\perp}^{2} / 4 \gamma} \int_{-\infty}^{+\infty} \mathrm{d} x_{\perp}^{2} \exp \left[i \gamma\left(x_{\perp}-\frac{q_{\perp}}{2 \gamma}\right)^{2}\right], \\
& =e^{-i q_{\perp}^{2} / 4 \gamma} \pi \int_{0}^{\infty} \mathrm{d} t e^{i \gamma t}
\end{aligned}
$$

on substituting $x_{\perp} \rightarrow x_{\perp}+q_{\perp} / 2 \gamma$ and using polar coordinates. Taking a contour in the first quadrant, we get

$L_{1}=e^{i \pi / 2} \frac{\pi}{\gamma} e^{-i q_{\perp}^{2} / 4 \gamma}$.

In the same way we can evaluate the integrals $L_{2}$ and $L_{3}$ by taking contours, respectively, in the fourth and first quadrants,

$$
\begin{aligned}
& L_{2}=e^{-i \pi / 4} \frac{\sqrt{\pi}}{\sqrt{\delta}} e^{i q_{0}^{2} / 4 \delta}, \\
& L_{3}=e^{i \pi / 4} \frac{\sqrt{\pi}}{\sqrt{\delta}} e^{-i q_{3}^{2} / 4 \delta} .
\end{aligned}
$$

Putting these values of integrals $L_{i}, i=1,2,3$, in (A.6) we get $\Lambda$ as given by Eq. (3.19) in the text.

\section{Appendix B: $\rho$-meson decay in vacuum}

As a consistency check on our method of calculation, here we apply it to recover the $\rho$-meson decay rate in vacuum. Consider the first term in $D$, behaving as $t^{-1}$. Integrating partially w.r.t. $v$, it gives 


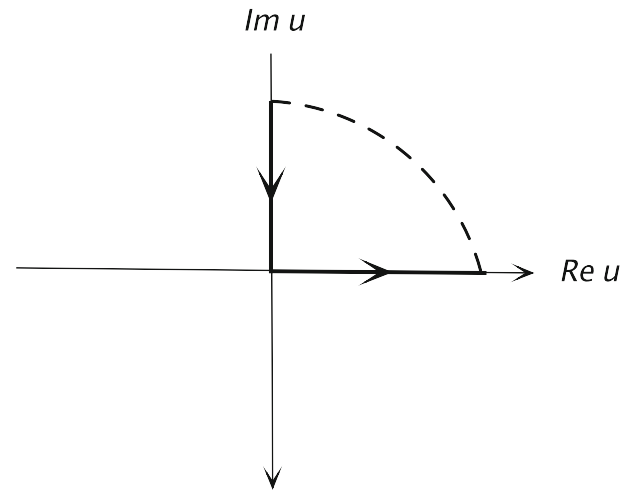

(a)

Fig. 2 Closed contour in $u$ plane in both first and fourth quadrants

$$
\begin{aligned}
& \left(\frac{g}{2 \pi}\right)^{2} \frac{q^{2}}{4} \int_{0}^{1} \mathrm{~d} v v^{2} \int_{0}^{\infty} \frac{\mathrm{d} t}{t} \exp \left[-\left\{m^{2}-\frac{q^{2}}{4}\left(1-v^{2}\right)\right\} t\right] \\
& =\left(\frac{g}{2 \pi}\right)^{2} \frac{q^{2}}{12}\left[\int_{0}^{\infty} \frac{\mathrm{d} t}{t} e^{-m^{2} t}+\frac{q^{2}}{2} \int_{0}^{1} \mathrm{~d} v v^{4}\right. \\
& \left.\quad \times \int_{0}^{\infty} \mathrm{d} t \exp \left[-\left\{m^{2}-\frac{q^{2}}{4}\left(1-v^{2}\right)\right\} t\right]\right]
\end{aligned}
$$

where the divergence at $t=0$ is isolated in the first term. Analogously the second term in $D$ behaving as $t^{-2}$ can also be put in a similar form after integrating twice partially w.r.t. $v$,

$$
\begin{aligned}
& \left(\frac{g}{2 \pi}\right)^{2} 2 \int_{0}^{1} \mathrm{~d} v \int_{0}^{\infty} \frac{\mathrm{d} t}{t^{2}} \exp \left[-\left\{m^{2}-\frac{q^{2}}{4}\left(1-v^{2}\right)\right\} t\right] \\
& =\left(\frac{g}{2 \pi}\right)^{2} 2\left[\int_{0}^{\infty} \frac{\mathrm{d} t}{t^{2}} e^{-m^{2} t}+\frac{q^{2}}{6} \int_{0}^{\infty} \frac{\mathrm{d} t}{t} e^{-m^{2} t}+\frac{q^{4}}{12} \int_{0}^{1} \mathrm{~d} v v^{4}\right. \\
& \left.\quad \times \int_{0}^{\infty} \mathrm{d} t \exp \left[-\left\{m^{2}-\frac{q^{2}}{4}\left(1-v^{2}\right)\right\} t\right]\right]
\end{aligned}
$$

Here also, the divergences at $t=0$ are isolated in the first two terms. These are the local divergent terms, which we expected in Sect. 3 for the general field case and which are analogous to those appearing in loop integrals over intermediate momenta in conventional field theory. Let us also note here that though individual terms in $\Lambda^{\prime}$ given by Eq. (3.22) do contain $(e B)^{2}$ dependent divergent terms, they cancel out in the complete expression for $\Lambda^{\prime}$, showing that divergences originate only from the vacuum piece of the self-energy, as expected. Leaving aside the divergent terms which will be absent in the imaginary parts, the $e B$ independent vacuum self-energy is given by combining the finite contributions from the two terms of $D$,

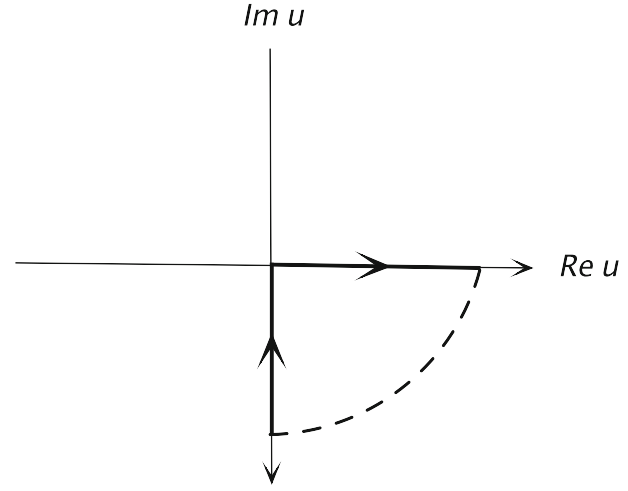

(b)

$$
\begin{aligned}
\Sigma_{0}\left(q^{2}\right)=- & \left(\frac{g}{2 \pi}\right)^{2} \frac{q^{4}}{8} \int_{0}^{1} \mathrm{~d} v v^{4} \int_{0}^{\infty} \mathrm{d} t \\
& \times \exp \left[-\left\{m^{2}-\frac{q^{2}}{4}\left(1-v^{2}\right)\right\} t\right] \\
= & -\left(\frac{g}{2 \pi}\right)^{2} \frac{q^{4}}{8} \int_{0}^{1} \mathrm{~d} v \frac{v^{4}}{m^{2}-q^{2}\left(1-v^{2}\right) / 4},
\end{aligned}
$$

where the $t$-integral is well defined within the limit $q^{2}<$ $4 m^{2}$. Now following an exactly similar procedure to that used in the $e B$ dependent self-energy part, we write $\Sigma_{0}\left(q^{2}\right)$ as a dispersion integral by changing variables $v=\sqrt{1-4 m^{2} / q^{\prime 2}}$ to $q^{\prime 2}$,

$$
\begin{aligned}
\Sigma_{0}\left(q^{2}\right)= & -\left(\frac{g}{2 \pi}\right)^{2} \frac{q^{4}}{4} \\
& \times \int_{4 m^{2}}^{\infty} \frac{\mathrm{d} q^{\prime 2}}{q^{\prime 2}}\left(\frac{1}{q^{\prime 2}-q^{2}}\right)\left(1-\frac{4 m^{2}}{q^{\prime 2}}\right)^{\frac{3}{2}} .
\end{aligned}
$$

This immediately allows us to write down the imaginary part of the vacuum part of the self-energy,

$\operatorname{Im} \Sigma_{0}\left(q^{2}\right)=-\frac{g^{2} q^{2}}{16 \pi}\left(1-\frac{4 m^{2}}{q^{2}}\right)^{\frac{3}{2}}$.

Now taking $q^{2}=m_{\rho}^{2}$, we find the standard decay rate in vacuum to be

$$
\begin{aligned}
\Gamma_{0} & =-\frac{\operatorname{Im} \Sigma_{0}\left(q^{2}\right)}{3 m_{\rho}} \\
& =\frac{g^{2} m_{\rho}}{48 \pi}\left(1-\frac{4 m^{2}}{m_{\rho}^{2}}\right)^{\frac{3}{2}},
\end{aligned}
$$

which when equated to the experimental rate would give $g=$ 6 as written at the beginning of Sect. 2 . 


\section{References}

1. C. Woltjer, Astrophys. J. 140, 1309 (1964)

2. W.A. Wheaton et al., Nature 289, 240 (1979)

3. I. Fushiki, E.H. Gudmundsson, C.J. Pethick, Astrophys. J. 342, 958 (1989)

4. D.E. Kharzeev, L.D. McLerran, H.J. Warringa, Nucl. Phys. A 803, 227 (2008)

5. V.V. Skokov, A.Yu. Illarionov, V.D. Toneev, Int. J. Mod. Phys. A 24, 5925 (2009)

6. A. Bzdak, V. Skokov, Phys. Rev. Lett. 110, 192301 (2013)

7. L. McLerran, V. Skokov, Nucl. Phys. A 929, 184 (2014)

8. K. Fukushima, H.J. Warringa, Phys. Rev. Lett. 100, 032007 (2008)

9. R. Gatto, M. Ruggieri, Phys. Rev. D. 82, 054027 (2010)

10. A. Ayala, M. Loewe, R. Zamora, Phys. Rev. D. 91, 016002 (2015)

11. K. Tuchin, Phys. Rev. C. 91, 064902 (2015)

12. A. Bandyopadhyay, C.A. Islam, M.G. Mustafa, Phys. Rev. D 94(11), 114034 (2016)

13. A. Bandyopadhyay, S. Mallik, Phys. Rev. D 95(7), 074019 (2017)

14. N. Sadooghi, F. Taghinavaz, Ann. Phys. 376, 218 (2017)

15. M.N. Chernodub, Phys. Rev. D 82, 085011 (2010)

16. H. Liu, L. Yu, M. Huang, Phys. Rev. D 91(1), 014017 (2015)
17. M. Kawaguchi, S. Matsuzaki, Eur. Phys. J. A 53(4), 68 (2017)

18. R. Zhang, Wj Fu, Yx Liu, Eur. Phys. J. C 76(6), 307 (2016)

19. S. Ghosh, A. Mukherjee, M. Mandal, S. Sarkar, P. Roy, Phys. Rev. D 94(9), 094043 (2016)

20. K. Fukushima, D.E. Kharzeev, H.J. Warringa, Phys. Rev. D 78, 074033 (2008)

21. D.E. Kharzeev, Ann. Phys. 325, 205 (2010)

22. V.A. Miransky, I.A. Shovkovy, Phys. Rep. 576, 1 (2015)

23. N. Mueller, J.A. Bonnet, C.S. Fischer, Phys. Rev. D 89, 094023 (2014)

24. V.P. Gusynin, V.A. Miransky, I.A. Shovkovy, Nucl. Phys. B 462 , 249 (1996)

25. J. Schwinger, Phys. Rev. 82, 664 (1951)

26. J. Schwinger, Particles, Sources and Fields, vol. I, II and III (Perseus Books, Reading, 1998)

27. J. Schwinger, Phys. Rev. 7, 1696 (1972)

28. A. Yildiz, Phys. Rev. 8, 429 (1973)

29. C. Itzykson, J.-B. Zuber, Quantum Field Theory (McGraw-Hill Book Company, New York, 1980)

30. R. Rapp, J. Wambach, Adv. Nucl. Phys. 25, 1 (2000)

31. V.I. Ritus, Ann. Phys. 69, 555 (1972) 